\title{
Novel parametric reduced order model for aeroengine blade dynamics
}

\author{
Jie Yuan', Giuliano Allegri ${ }^{1,4}$, Fabrizio Scarpa ${ }^{1,3^{*}}$, Ramesh Rajasekaran ${ }^{2}$, Sophoclis Patsias ${ }^{2}$ \\ ${ }^{1}$ Advanced Composites Centre for Innovation and Science, University of Bristol, BS8 1TR Bristol, UK \\ ${ }^{2}$ Mechanical Methods, Rolls-Royce plc, PO Box 31, DE24 8BJ Derby, UK \\ ${ }^{3}$ Dynamics and Control Research Group (DCRG), University of Bristol, BS8 1TR Bristol, UK \\ ${ }^{4}$ Department of Aeronautics, Imperial College London, London SW7 2AZ, UK
}

\begin{abstract}
The work introduces a novel reduced order model (ROM) technique to describe the dynamic behavior of turbofan aeroengine blades. We introduce an equivalent 3D frame model to describe the coupled flexural/torsional mode shapes, with their relevant natural frequencies and associated modal masses. The frame configurations are identified through a structural identification approach based on a simulated annealing algorithm with stochastic tunneling. The cost functions are constituted by linear combinations of relative errors associated to the resonance frequencies, the individual modal assurance criteria (MAC), and on either overall static or modal masses. When static masses are considered the optimized 3D frame can represent the blade dynamic behavior with an $8 \%$ error on the MAC, a $1 \%$ error on the associated modal frequencies and a $1 \%$ error on the overall static mass. When using modal masses in the cost function the performance of the ROM is similar, but the overall error increases to $7 \%$. The approach proposed in this paper is considerably more accurate than state-of-the-art blade ROMs based on traditional Timoshenko beams, and provides excellent accuracy at reduced computational time when compared against high fidelity FE models. A sensitivity analysis shows that the proposed model can adequately predict the global trends of the variations of the natural frequencies when lumped masses are used for mistuning analysis. The proposed ROM also follows extremely closely the sensitivity of the high fidelity finite element models when the material parameters are used in the sensitivity.
\end{abstract}

Keywords: beam frame, turbofan blades, mistuning analysis

\begin{tabular}{|l|l|}
\hline$A_{i}$ & Nomenclature \\
\hline$E_{i}$ & Area of $\mathrm{i}^{\text {th }}$ element \\
\hline$L_{i}$ & Kinetic energy value for the $\mathrm{i}^{\text {th }}$ mode in Finite Element (FE) model \\
\hline $\boldsymbol{K}_{\boldsymbol{m} m}, \boldsymbol{K}_{\boldsymbol{s s}}, \boldsymbol{K}_{\boldsymbol{s m}}$ & Length of $\mathrm{i}^{\text {th }}$ element \\
\hline $\boldsymbol{M}, \boldsymbol{K}$ & Stiffness matrix in the master, slave and coupled degree of freedoms \\
\hline $\boldsymbol{M}_{\boldsymbol{R}}, \boldsymbol{K}_{\boldsymbol{R}}$ & Full-scale mass and stiffness matrix \\
\hline $\boldsymbol{M}_{\boldsymbol{m} \boldsymbol{m}}, \boldsymbol{M}_{\boldsymbol{s s}}, \boldsymbol{M}_{\boldsymbol{s m}}$ & Reduced mass and stiffness matrices \\
\hline$M_{a}, M_{F E}$ & $\begin{array}{l}\text { Mass matrix in the master (mm), slave (ss) and coupled (sm) degree of } \\
\text { freedoms }\end{array}$ \\
\hline$M_{i}^{F E}$ & Total mass in the analytical model (a) and FE model (FE) \\
\hline$M_{i}^{a}$ & Modal mass value in FE model for $\mathrm{i}^{\text {th }}$ mode \\
\hline $\bar{M}_{i}^{F E}$ & Modal mass value in analytical model for $\mathrm{i}^{\text {th }}$ mode \\
\hline $\bar{M}_{i}^{a}$ & Relative modal mass in FE model \\
\hline
\end{tabular}

${ }^{*}$ Corresponding author. Tel.: +44(0)1173315306

E-mail address: F.scarpa@bristol.ac.uk 


\begin{tabular}{|l|l|}
\hline $\boldsymbol{T}_{I}, \boldsymbol{T}_{S}$ & Transformation matrix and static Transformation matrix \\
\hline & \\
\hline$m_{a}$ & Global mass matrix of analytic model \\
\hline$m_{i}^{G}, k_{i}^{G}$ & Global coordinate mass and stiffness matrices of the $\mathrm{i}^{\text {th }}$ element \\
\hline $\bar{m}_{i}^{G}, \bar{k}_{i}^{G}$ & $m_{i}^{G}, k_{i}^{G}$ in an assembled size matrix \\
\hline$I_{x i}$ & Polar moment of area for the $\mathrm{i}^{\text {th }}$ element \\
\hline$I_{y i}, I_{z i}$ & Second moment of area around y and $\mathrm{z}$ direction for the $\mathrm{i}^{\text {th }}$ element \\
\hline$w_{i}$ & Weight of the $\mathrm{i}^{\text {th }}$ natural frequency in the objective function \\
\hline$\alpha_{i}$ & Coefficient of $A_{i}$ in structure identification equation \\
\hline$\beta_{i}$ & Coefficient of $I_{x i}$ in structure identification equation \\
\hline$\gamma_{i}$ & Coefficient of $A_{i}$ in structure identification equation \\
\hline$\delta_{i}$ & Coefficient of $I_{y i}$ in structure identification equation \\
\hline$\zeta_{i}$ & Coefficient of $I_{z i}$ in structure identification equation \\
\hline$\eta_{i}$ & Coefficient of $I_{x i}$ in structure identification equation \\
\hline$\Phi$ & Assembled mode shapes \\
\hline$\phi_{i}^{r}$ & r $^{\text {th }}$ mode shape for the ith element \\
\hline$\phi_{m}, \phi_{s}$ & Master (m) and slave(s) modal coordinates \\
\hline$\omega_{i}$ & $\mathrm{i}^{\text {th }}$ natural frequency from FE model \\
\hline$\omega_{i}^{a}$ & $\mathrm{i}^{\text {th }}$ natural frequency from analytical model \\
\hline$\delta_{r s}$ & Kronecker delta function \\
\hline
\end{tabular}

\section{Introduction}

A bladed disk typically consists of a set of disk/blade sectors that are designed to be identical. However, there are always small variations in the structural properties of individual blades due to manufacturing and assembly tolerances, material imperfections and operational wear and tear [1]. These dimensional and material uncertainties lead to variations of the blade natural frequencies from their nominal design value. This phenomenon is generally denoted as blade mistuning. Mistuning in the free response splits the repeated natural frequencies associated with circumferential modes and distorts the corresponding mode shapes [2-6]. Simultaneously, these circumferential mode shapes increase the harmonic content of nodal diameters, leading to coupling with engine-induced vibrations [7]. In the worst case scenario mistuning also causes mode localization phenomena, in which the vibrational energy is transferred and confined to a subset of blades. This phenomenon may result in dynamic deformations significantly larger than those estimated at the design stage $[8,9]$. As a consequence, mistuning compromises the high-cycle fatigue endurance of bladed disks and reduces the durability and the reliability of the entire engine. As a result, the research on the attenuation of mistuning has been a key activity in aero-engine R\&D for more than 50 years, although the mistuning problem is considered still unsolved [2].

High-fidelity finite element models of bladed disks are used to predict both the maximum dynamic excitation at representative design points and the associated behavior due to pre-defined mistuning patterns $[10,11]$. From a general perspective, analytical and numerical models of mistuning are more cost-effective than direct experimental characterizations because a large number of tests on nominally identical bladed disks are necessary to have a statistically representative population for all the various sources of uncertainty leading to mistuning. Since mistuning breaks the cyclic symmetry of bladed-disk systems, [7], simplified FE models of single sectors are not suitable for analysis purposes and in most cases a full bladed disk model is necessary. A complete FE model of a bladed disk however typically 
involves the use of millions of degrees of freedom (DOFs), making parametric analyses too expensive even on state-of-the-art high-performance computing facilities. The potential use of probabilistic approaches (i.e., Monte Carlo simulations, even by using improved techniques of sampling generation such as Latin hypercube) further increases the computational costs. As a consequence, various FE-based reduced order methods (ROM) have been developed in the last two decades.

ROMs used in mistuning applications can be broadly classified into two groups, which are both based on modal reduction techniques. The first group developed between 1983 and 2000 consists essentially in Component Mode Synthesis (CMS) techniques [12-14]. CMS approaches assume that the blades and the disk are distinct sub-structural elements, thus their modes are separately calculated via deterministic FE analyses. The modal bases of the two substructures are then employed to reduce the size of the overall system matrices by enforcing compatibility conditions at the interfaces between the blades and the disk. The compatibility between the dynamic displacements existing within the frontiers of substructures can be described by means of fixed-interface [15], free-interface [12] and hybrid-interface methods [13]. The type of interface used affects the numerical convergence of the ROM in a significant way. Fixed interface can exhibit very good convergence properties by increasing the number of component modes, but it requires large numbers of interface DOFs and therefore penalizes the computational efficiency[16]. Another limitation of the fixed interface method is the inability to obtain easily the required constrained modes from testing, making this method used primarily in analytical or purely numerical models [17]. Free interface methods have a slow convergence, although in principle this problem can be overcome by using residual flexibility (attachment modes) provided by low frequency approximations to describe the contribution from neglected high frequency modes [16]. Unfortunately, it is difficult to extract accurate residual flexibility terms from test data, as well as information on residuals in analytical model [17]. The second group of approaches initially introduced by Yang and Griffin in 2001 [18] are denoted as System Mode based Methods (SMM). These include the Fundamental Mistuning Model [19], the Component Mode Mistuning [20] and the Integral Mode Mistuning [2]. The main concept behind SMM techniques is to employ selected sets of tuned system modes as a basis to represent the tuned disk-blade system, and then add a perturbation matrix to represent the mistuning effect. This strategy in principle allows an exact representation of the baseline tuned system, and SMM techniques are also more computationally efficient than CMS approaches. The accuracy of the SMM is very much dependent on the modal representation of the tuned system, and practice in the industry has revealed the use of SMM as reliable predictive tool for the first six mistuned modes, which involves the extraction of hundreds of modes from the FE full-scale models. CMS and SMM models are moreover not able to perform the mistuning analysis by direct perturbation of specific parameters in the modal domain, but they generally introduce the mistuning in the form of a natural frequency deviation, which complicates the sensitivity studies required for robust design. Most importantly in sensitivity analyses, all these ROMs have to be rebuilt or updated through a repeated extraction of modes from perturbed FE models to guarantee accurate predictions, thus reducing the computational efficiency. Current state-of-the-art parametric analyses for mistuning problems often employ lumped parameter approaches. Although these models can reproduce some of the basic dynamics characteristics of bladed disk systems and can be used for the statistical analysis of mistuning forcedresponse behavior, their practical applicability is limited at best to the first three modes [7]. In terms of classical reduction techniques used in the industry, the accuracy provided by Improved Reduced System (IRS) techniques largely depends on the selection of numbers and locations of the master degree of freedoms (MDOFs) [21], as in classical Guyan reduction techniques[22]. Moreover, they are not able to exploit the cyclic nature of the geometry of bladed disc systems.

A consistent body of literature has also been devoted to investigate the effects generated by uncertainties associated to the geometry or material properties on the dynamics of the blade alone [1]. The blade 
models used in these works are mainly based on high-fidelity FE models, or coordinate measurement machine data $[1,23,24]$. State-of-the-art in ROMs to represent single rotating blades mainly consists in using simple cantilever beams with rectangular cross section to approximate the exact shape by finite element representation $[25,26]$. Torsion-induced displacement are present in the vibrational patterns of these blades, and they are mainly induced by the coupling between the bending deformations in the flap wise and chord wise directions. Second order effects (like shear deformations, rotary inertia, warping of the cross section, root fixing and Coriolis accelerations) do also contribute in providing a complex dynamic displacement pattern. Fan and compressor blades have been traditionally modeled using either simple twisted Euler-Bernoulli's beam approaches, or twisted Timoshenko's beam formulation with varying levels of complexity and warping $[27,28]$. Rotating pre-twisted blade dynamics for the two types of structural beams has also been thoroughly investigated [28]. Although these simplified models are able to capture the first few fundamental modes (global bending, torsion, axial stretching and coupled bending motions in two directions), they are less suitable to represent the mode shapes of real blades, characterised by strongly coupled bending and torsional deformations, chord wise bending and edge wise flap. Designers in the field have also pointed out that beam models provide a poor performance when it is required to predict the blade modes associated with chord wise bending, i.e. second strip mode [27]. The uncertainty propagation analysis of these models is mainly restricted to the material properties and lumped mass at particular positions for the first few global modes.

This paper addresses the development of a parametric reduced order model of a blade using a novel approach consisting in introducing a simplified structural layout (frame structure) that provides a dynamic behavior equivalent to that of the full-scale blade. The ROM model developed in this paper is primarily meant to be employed in sensitivity analyses, where the uncertainties associated to either blades from material properties' deviation and geometrical mistuning or the joints due to manufacturing, assembly tolerances and blade/disk loosening during the high speed rotation are considered [29]. As a case study, we consider a metallic fan blade described using a "high-fidelity" (HF) solid FE modelling that provides the baseline characterization of the first six global modes. The ROM frame model is developed through a structural identification approach based on a simulated annealing algorithm with stochastic tunneling, and also benchmarked against an equivalent Timoshenko beam ROM as a reference. The equivalent frame concept can include either 2D or 3D beam kinematics. An optimization process involving the geometric configuration of the frame (ROM) model is then introduced to minimize the natural frequency (NF), MAC and overall mass/modal mass errors with respect to the full blade FE model. The identification of the ROM parameters is carried out with two different cost functions. The first includes the total static mass of the blade, while the second consider the modal masses [30, 31]. While the objective that involves the total static mass reflects a physical equivalence between the ROM and the baseline blade model, the use of the modal masses is representative of the effective inertia associated to each mode. As it will be shown, the results of the ROMs optimised according to the two different sets of objectives do differ and the static mass based optimisation leads to better results than those associated with the modal masses. This indicates that the identification of the ROM parameters via optimization procedures should consider the total static mass as an objective function in order to minimise the errors associated to natural frequencies and Modal Assurance Criteria (MAC). Finally, a comparison of the sensitivity performance of the ROM beam frame and the high fidelity FE model with lumped masses and perturbation of the material parameters is carried out to validate the new ROM concept for mistuning applications, with uncertainty distributions associated to the blade.

\section{Reference blade model}

The HF reference FE model of the blade is built using the commercial software ANSYS Rel. 11.0 (Ansys 
Inc., 2008) (Figure 1). The model consists in 540 SOLID95 8-node elements. Each node has three translational DOFs. The total number of DOFs is 32400. The blade is entirely made of Titanium, considered as a homogenous and isotropic material. The blade has a low slenderness ratio, i.e. 4 , and an increasing twist from root to tip.

The HF model is clamped at the root by zeroing all DOFs. A modal analysis has been performed on the HF model using a Block Lanczos method. All the simulations described in this paper, both full FE and ROM, have been carried out on a PC, with an Intel 3.2 GHz dual-core processor and 4 GB RAM. The NFs and modal displacements in global Cartesian axes are shown in Figure 2 for the first six modes. The relative spacing of the first six natural frequencies is $1: 2.8: 3.9: 6.5: 8.5: 9.9$. It can be observed that out-ofplane bending, torsion and edge-wise out-of-plane flapping dominate the first six modes. In-plane bending and axial stretching do not appear in the first six modes. While the first mode essentially consists in out-of-plane bending, the following four modal shapes are characterised by coupled torsion and bending deformations. An edgewise flap motion dominates the sixth mode. Moreover, for the first six modes the average span-wise modal displacement is about ten times smaller than the one along the outof-plane direction. Although out-of-plane deformations dominate the low order modes, significant deformations still appear along the in-plane chord-wise direction, especially close to the blade tip. This is due to the geometric twist of the structure. All six modes exhibit a high level of asymmetry along the chordwise direction.

\section{Classical Timoshenko beam ROM approach}

The Timoshenko beam approach is applied to the blade considered in this work because the length to width ratio is smaller than five, and it is therefore necessary to include the effects of shear deformation and rotatory inertial to the structure. A single and straight Timoshenko beam model with three elements is applied, with its relevant stiffness and mass matrices derived from standard open literature. The material properties are assumed as the ones of titanium (Young's modulus of $1.15 \mathrm{e} 5 \mathrm{Mpa}$, density of $4430 \mathrm{~kg} / \mathrm{m}^{3}$ and Poisson's ratio of 0.32). The lengths of three elements are the same as ones of the novel frame concept proposed in the next section. The moments of inertias along the three directions and areas of the cross sections are estimated from the HF FE model. A clamped-free modal analysis of the ROM is then performed using an eigenvalue extraction based on QR method (function eig) using Matlab R2012a. The NF and type of mode shape for both the HF and the Timoshenko ROM model are described in Tables 1 and 2 .

The average NF error for the first three modes is $10 \%$, while the one related to the last three natural frequencies is significantly larger, reaching almost 50\%. Table 2 shows the mode shapes for the first six modes extracted from the Timoshenko beam ROM blade. The first three modes are flapwise bending, torsion and second flapwise bending, which are close to the global modes in the twisted blade shown in Figure 1. However, the twisted blade (HF model) does not show three pure global mode shapes as the beam model, but modes with high bending-torsion coupling, which makes therefore the use of the Timoshenko beam ROM difficult to justify. As a further testimony of this fact, the last three NF errors are significantly larger than the ones related to the first three modes.

\section{The frame-based approach}

The ROM proposed in this paper is based on a frame consisting of 9 individual beams. The rationale for this choice is justified by the fact that the geometry of each individual beam can be tailored to represent complex mode shapes, e.g. those for which bending and twisting are coupled. Moreover, a frame retains a 
sufficient level of geometrical similarity with the original blade. This fact allows a direct mapping of the displacements at prescribed locations on the blade to points belonging to the frame. This mapping facilitates the ROM calibration process based on displacement data obtained from the HF FE blade model.

To represent the out-of-plane motion of the airfoil, a frame whose elements are 2D Euler-Bernoulli beams has been considered first. This particular frame constitutes a reasonable approximation of the blade, since the first six actual modes are dominated by out-of-plane displacements, while in-plane stretching and bending affect only higher order modes. Using 2D Euler-Bernoulli beams for the ROM, each node has three DOFs (i.e. Uz, Rotx, Roty), as shown in Figure 4 (a). Albeit this model allows the representation of out-of-plane bending, global twist and span-wise flapping, the coupled displacements and rotations associated with the chord-wise deformation are neglected. Therefore, a frame consisting of 3D EulerBernoulli beams (i.e. 6 dofs per node) is also considered and its performance assessed with respect to that of the 2D model. For both the 2D and 3D ROM frames, the number of nodes must be sufficient to ensure an adequate representation of the selected modes, otherwise spatial aliasing occurs (Fotsch and Ewins, 2000).

The mass and stiffness matrix of single 2D and 3D Euler-Bernoulli beam elements and the transformation matrices from local to global coordinates are available in the literature [32] and will be directly used herein. The full frame models are assembled from the beam elements using the MATLAB software. These models are parametric; the cross-section of each beam can be varied in order to calibrate the ROMs, while the material properties are the same for both the ROMs and the HF FE model. The assumed material properties are those characteristic of titanium (Young's modulus of $115 \mathrm{GPa}$, density of $4430 \mathrm{~kg} / \mathrm{m}^{3}$ and Poisson ratio of 0.32). Each beam within the frame is assumed to have a symmetric cross section, uniform along the length. The cross sectional areas and second moment of areas are the variables that are updated for the ROMs calibration. A single finite element is employed for each beam in the frame. The length of each element is kept constant during calibration.

The auto MAC is a statistical indicator of the autocorrelation between mode shapes obtained with different analysis and/or experimental methods [33]. The auto MAC is here employed to determine whether the ROMs are sufficiently accurate in capturing the mode shapes obtained from the HF FE analysis. Clearly, the same comparison could have been established between the ROMs and experimental data, if those were available. However, the emphasis is here placed on introducing a reduced order modeling approach based on the frame structure shown in Figure 4 and the validation of this technique by means of experimental data will be carried out in future work.

Before starting calibrating the reduced-order model, it is necessary to assess whether the displacements extracted from the full blade FE model are sufficient to identify the dynamics of the structure. The first six modes of the blades are considered and the auto MACs are computed for sets of respectively four, six and eight nodes located on both the edges of the HF FE model (figure 5). The associated results are plotted in Figure 5. It can be observed that using four measurement points yields poor MAC results, since the corresponding off-diagonal terms give cross-correlation coefficients close to 1 . This is a spurious correlation, which is due to the too low number of measurement points considered. Increasing the number of measurement points to 6 and later to 8 progressively reduces the magnitude of the off-axis crosscorrelation terms. Most of the off-diagonal corrections in the 8 nodes plot approach to zero with maximum MAC value of 0.3 . Some modes are still not completely linearly independent; however the first six modes can be easily distinguished from each other. In open literature [15, 34], it has been demonstrated that 8 measurement points are sufficient to identify the first 6 modes, provided that the maximum MAC value for off-diagonal terms is below 0.3 . These conditions are met by the node subset 
considered here.

\section{Initial value determination}

In order to accelerate the ROM calibration, it is necessary to identify a "suitable" initial solution. This implies finding an initial reasonable guess for the cross-sectional parameters featuring the ROM beams. This could be done, for example, by imposing the equivalence of the total elastic potential and kinetic energy between the ROMs and the full HF FE model [35]. However, this equivalence requires the use of specific numerical models as the source of these data, therefore it is not directly applicable to cases where only experimental results are available. Classical modal updating techniques, either direct methods or iterative methods, are also not suitable since they require a detailed and relatively accurate initial model, with good correlations between analytical and experimental results[36]. An approach recently developed by Karaağaçlı et al. [36]allows overcoming the limitations of traditional updating techniques. This method only requires data from experimental modal analysis. It provides a simple and fast way to determine the initial ROM configuration, albeit issues related with the ill-conditioning of structural identification matrices may arise. We hereby describe how the method is applied in the context of our analysis.

\section{Step one: Expansion of the mode shapes}

Since the values of the rotational DOFs in the measuring points are not available either from the experiment or via HF FE models (as in the present case), the mode shapes can be reconstructed using a Inversed Improved Reduced System (IRS) expansion technique based on a static reduction method [37]. The expansion is provided through the modal basis from the "experiment", i.e. the HF FE model in our case.

The modal displacement associated with the slave DOFs $\phi_{s}$ can be expanded by using the master mode shape $\phi_{m}$ and the transformation matrix $\boldsymbol{T}_{\boldsymbol{I}}$ :

$\left\{\begin{array}{c}\phi_{m} \\ \phi_{s}\end{array}\right\}=T_{I} \phi_{m}$

In the present case, the translational displacements for the first six modes provide the master mode shape $\boldsymbol{\phi}_{\boldsymbol{m}}$. The transformation matrix $\boldsymbol{T}_{\boldsymbol{I}}$, which includes the inertia terms as pseudo static forces, is evaluated as:

$T_{I}=T_{S}+S M T_{S} M_{R}^{-1} K_{R}$

In Eq. (2), $T_{S}$ is a static transformation matrix, while $M_{R}, K_{R}$ are mass and stiffness matrices associated with the slave DOFs. These matrices are given by:

$T_{S}=\left[\begin{array}{c}I \\ -K_{s s}{ }^{-1} K_{s m}\end{array}\right], \quad S=\left[\begin{array}{cc}0 & 0 \\ 0 & K_{s s}{ }^{-1}\end{array}\right], M_{R}=T_{S}^{T} M T_{s}, K_{R}=T_{S}^{T} K T_{s}$

In Eq. (3) $M$ and $K$ are the matrixes of the complete dynamic system, i.e. those featuring both translational and rotational DOFs. In order to apply the procedure outlined above, the complete mass and stiffness matrixes need to be reordered and partitioned into slave $(s)$ and master $(m)$ DOFs: 
$M=\left[\begin{array}{cc}M_{m m} & M_{m s} \\ M_{s m} & M_{s s}\end{array}\right], \quad K=\left[\begin{array}{cc}K_{m m} & K_{m s} \\ K_{s m} & K_{s s}\end{array}\right]$

\section{Step two: Structure identification}

The identified mode shapes (including their rotational DOFs) satisfy the mass and stiffness orthogonality conditions:

$\left[\Phi^{T}\right] \sum_{i=1}^{9} \overline{\boldsymbol{m}}_{\boldsymbol{i}}^{G}[\Phi]=[I]$

$\left[\Phi^{T}\right] \sum_{i=1}^{9} \overline{\boldsymbol{k}}_{\boldsymbol{i}}^{\boldsymbol{G}}[\Phi]=\left[\because \omega_{r}^{2} \cdot\right]$

In Eqs. (5) and (6) the global mass and stiffness matrices are represented by the sum of the partitioned element matrixes $\bar{m}_{i}^{G}, \bar{k}_{i}^{G}$ in global coordinates. The modal displacements of the $r^{\text {th }}$ mode are mapped to each frame element as $\phi_{1}^{r}, \phi_{2}^{r}, \ldots \phi_{9}^{r}$ (the frame is composed by nine elements, as shown in fig. 4). Eqs. (5) and (6) are therefore further decomposed according to the modal expansions associated to each beam element of the frame:

$\left\{\phi_{1}^{r}\right\}^{T}\left[m_{1}^{G}\right]\left\{\phi_{1}^{S}\right\}+\left\{\phi_{2}^{r}\right\}^{T}\left[m_{2}^{G}\right]\left\{\phi_{2}^{S}\right\}+\cdots\left\{\phi_{9}^{r}\right\}^{T}\left[m_{9}^{G}\right]\left\{\phi_{9}^{S}\right\}=\delta_{r s}$

$\left\{\phi_{1}^{r}\right\}^{T}\left[k_{1}^{G}\right]\left\{\phi_{1}^{S}\right\}+\left\{\phi_{2}^{r}\right\}^{T}\left[k_{2}^{G}\right]\left\{\phi_{2}^{S}\right\}+\cdots\left\{\phi_{9}^{r}\right\}^{T}\left[k_{9}^{G}\right]\left\{\phi_{9}^{S}\right\}=\omega_{r}^{2} \delta_{r s}$

From Eqs. (7) and (8), a series of equations with the unknown geometry parameters of the 3D beam frame can be derived by substituting the mass and stiffness matrices of a single beam element $m_{i}^{G}, k_{i}^{G}$ :

$\alpha_{1}^{r s} A_{1}+\beta_{1}^{r s} I_{x 1}+\alpha_{2}^{r s} A_{2}+\beta_{2}^{r s} I_{x 2}+\cdots \alpha_{9}^{r s} A_{9}+\beta_{9}^{r s} I_{x 9}=\delta_{r s}$

$\gamma_{1}^{r s} A_{1}+\delta_{1}^{r s} I_{y 1}+\zeta_{1}^{r s} I_{z 1}+\eta_{1}^{r s} I_{x 1}+\gamma_{2}^{r s} A_{2}+\delta_{2}^{r s} I_{y 2}+\zeta_{2}^{r s} I_{z 2}+\eta_{2}^{r s} I_{x 2}+\cdots$

$+\gamma_{9}^{r s} A_{9}+\delta_{9}^{r s} I_{y 9}+\zeta_{9}^{r s} I_{z 9}+\eta_{9}^{r s} I_{x 9}=\omega_{r}^{2} \delta_{r s}$

In Eqs. (9) and (10), $\alpha^{r s}, \beta^{r s}, \gamma^{r s}, \delta^{r s}, \zeta^{r s}, \eta^{r s}$ are parametric coefficients derived from the orthogonality between the $\gamma^{\text {th }}$ and $s^{\text {th }}$ modes. Considering 6 modes, 21 equations of the type (9) and (10) can be established. A further equation can be imposed on the total mass of the beam frame:

$\rho A_{1} L_{1}+\rho A_{2} L_{2}+\cdots \rho A_{9} L_{9}=M_{F E}$

Eq. (11) is applied if the structural identification is carried out under the constraints that the frame mass must equal that of the full HF FE model.

Alternatively, one can consider a constraint for the ROM involving the modal masses. The modal mass matrix can be mathematically defined as a diagonal matrix when the mass matrix is post-multiplied by the mode shape matrix and pre-multiplied by its transpose [30]. The mass matrix is generally unknown in the experiments. In principle it is possible to extract the mass matrix directly from the FE models, albeit its manipulation to evaluate modal masses is computationally expensive [38]. An experimental methodology to extract modal masses has been proposed by Allemang et. al. [31]. In term of FE models, a kinetic energy approach is widely used as an effective method to extract modal masses [38], which can be calculated as follows: 
$M_{i}^{F E}=2 E_{i} / \omega_{i}^{2}$

The modal masses $M_{i}$ of the ROM can be directly evaluated through the available global mass matrix with the modal eigenvectors normalized with respect to the maximum generalized displacement:

$M_{i}=\phi_{i}^{T} m_{a} \phi_{i}$

When equating Eqs. (13) and (12), one can specify a constraint on the modal masses for the ROM.

\section{Step three: Limit ill-conditioning and solve the equations}

In the procedure detailed above, ill-conditioning problems may arise due to the approximations inherent to the modal expansion and the different order of magnitudes of the unknown geometrical parameters. However, the geometrical parameters that provide a negligible contribution to the modal strain energy in Eq. (8) can be safely disregarded [39]. In the present case the span-wise extensional deformations can be neglected. Moreover, the two beams connected to the base are assumed to have the same stiffness and cross-sectional dimensions in order to reduce the rank deficiency of the systems in Eqs. (9-11) or in Eqs. $(9-10,12)$. The "experimental" NFs are used in the structural identification equation (9-10).

\section{Optimization process}

The optimization process is carried out starting from the initial configuration of the frame identified with the IRS technique described above. The cost function consists of the linear combination of three objective functions:

Cost function $=\sum_{i=1}^{3} w_{i} O b j_{i}$

The first two objectives are the relative error on the NF and the MAC between the ROMs and the HF FE model:

$$
\begin{aligned}
& O b j_{1}=\sqrt{\sum_{i=1}^{6}\left\{\left(p_{i}\left(\omega_{i}^{a}-\omega_{i}\right) / \omega_{i}\right)^{2}\right\}} \\
& O b j_{2}=\sqrt{\sum_{i=1}^{6}(1-M A C(i))^{2}}
\end{aligned}
$$

The $p_{i}$ factors in equation (15) represent additional weights. Since the NF tends due increase with the rotational speed due to the stress stiffening effect (Ewins, 2010), the associated weight $\mathrm{p}_{1}$ has been arbitrarily set to 1.5 , while $p_{i}=1$ for $i=2,3, \ldots, 6$. The increase of $p_{1}$ does enhance the sensitivity of the objective function to the relative error in the first NF, and allows avoiding possible intersections with other fundamental engine harmonics in the Campbell diagram. All the geometric variables and eigenvalues are restricted to be real and positive.

For the third objective function, we consider two different scenarios. As a first case, we consider the relative error on the total mass of the ROM $\mathrm{M}_{\mathrm{a}}$ and that of the full HF FE model $\mathrm{M}_{\mathrm{o}}$ :

$O b j_{3}=\left|\left(M_{a}-M_{o}\right) / M_{o}\right|$

As a second case, we consider the error on the relative modal masses between the ROM and the HF FE: 
$\mathrm{Obj}_{3}=\sqrt{\sum_{i=1}^{6}\left(\bar{M}_{i}^{F E}-\bar{M}_{i}^{a}\right)^{2}}$

The relative modal masses are separately calculated as:

$\bar{M}_{i}^{F E}=M_{i}^{F E} / M_{F E}$

$\bar{M}_{i}^{a}=M_{i}^{a} / M_{a}$

The piecewise linear surface responses of each $\mathrm{NF}$ as a function of the geometrical variables are shown in Figure 6. Although the surfaces are smooth, the cost function defined in Eq. (14) has 21 independent variables and therefore it presents a significant number of local minima. This makes convex gradientbased optimization methods unsuitable for calibrating the ROM. In the present case, stochastic global optimizers like simulated annealing (SA) and genetic algorithm (GA) are best suited for the purpose of calibrating the ROM. In principle, GA and SA provide similar performance in dynamic structural identification problems [40], albeit GA tend to be more computationally expensive. However, traditional SA techniques may converge to local minima if the potential energy difference among adjacent troughs is much smaller than the energy required for a state transition [41]. This issue can be prevented by coupling SA with "Stochastic Tunneling" (ST) [41]. The latter is based on a nonlinear transformation of the energy landscape. In this work, a coupled SA-ST optimization algorithm is employed. The potential energy surface in SA is explored via a random walk, where the transition from a given configuration with energy $E_{1}$ to a new configuration with energy $E_{2}$ takes place with probability $p$ given by:

$$
p=\exp \left(\beta\left(E_{2}-E_{1}\right)\right), \beta=\alpha N
$$

where $\beta$ is inverse fictious temperature represented by iterative number $\mathrm{N}$ and constant coefficient $\alpha$. In the case of the SA-ST algorithm, the potential energy surface is dynamically transformed as follows:

$$
E_{\text {stun }}(x)=1-\exp \left(-\gamma\left(E(x)-E_{o}\right)\right)
$$

where $\gamma$ is a tunnelling parameter and $\mathrm{E}_{\mathrm{o}}$ is lowest minimum encountered during the random walk.

\section{Optimization with static mass constraint - results and discussions}

Figures $7 \mathrm{a}$ and $7 \mathrm{~b}$ show the Pareto fronts of the 2D and 3D ROMS extracted from 40000 feasible solutions. The colored map expresses the values of the static mass errors. CPU times are also indicated. The optimal solutions cluster near the Pareto front. This shows the sensitivity to different combinations of NF and MAC errors, depending on the weight ratio imposed in the cost function. The two models provide similar performance for a total mass error below $1 \%$. The location of the Pareto front in the 2D case is mainly within the region between 0.7 and 0.85 for $\mathrm{Obj} 2$, and from 0.1 to 0.4 for $\mathrm{Obj} 1$. For the $3 \mathrm{D}$ frame the intervals defining the Pareto front are different, with Obj2 ranging from 0.17 to 0.18 and $\mathrm{Obj} 1$ bounded between 0.005 and 0.05 . These results suggest that the 3D ROM outperforms the 2D model in terms of NF and MAC errors. However, the computational time required for the $3 \mathrm{D}$ ROM is 3.5 times that spent for calibrating the $2 \mathrm{D}$ frame.

The 2D and 3D frame models are further compared at the design point represented by the optimal solution with weights equal to 2:2:1 and 2:7:1 respectively. The requirements in these configurations are for relative errors of the NFs being less than 1\%, and MAC errors lower than $10 \%$ for each of the first six modes. As long as these conditions are satisfied all the configurations will be regarded being feasible, which will also facilitate the model parameterization. The weights ratios are selected from the feasible solutions with the lowest values of the cost function. The user can further filter the configurations based 
on specific targets and constraints.

The relative NF and MAC errors of the first six modes in the 2D and 3D frame ROMs are plotted against the initial errors in Figures $8 \mathrm{a}$ and $8 \mathrm{~b}$ respectively. The SA-ST optimizer significantly reduces the initial errors for both the ROMs. The average NF error is $6 \%$ for the $2 \mathrm{D}$ frame and $1 \%$ for the 3D ROM. The 2D frame yields a MAC error (30\% average) that is substantially higher than the $8 \%$ observed for the $3 \mathrm{D}$ ROM case. However, the use of SA has allowed the NF error associated to the $2 \mathrm{D}$ frame case to decrease $25 \%$ from the initial identification. The superior performance of the $3 \mathrm{D}$ beam frame is justified by the intrinsic coupling between in-plane deformations and out-of-plane torsion existing in the ROM, which increases the accuracy of the optimization process in identifying the beam parameters. Albeit the optimization process based on the 2D frame is 4 times faster than that of the 3D ROM, the associated errors are significantly larger. MAC comparisons between the ROM models and the HF FE for both the 2D and 3D cases are shown in Figure 9. Weak correlations can be observed for most the off-diagonal elements for the 3D ROM, with peak values of 0.3 for modes 4 and 2 and modes 5 and 6 . The offdiagonal MAC values obtained from the 3D ROM are very close to those evaluated from the HF-FE model. Thus, the high correlations between modes 5 and 6 and modes 4 and 2 can be only attributed to spatial aliasing. As expected, the optimized 3D ROM outperforms the 2D frame in term of off-diagonal MAC correlations.

\section{Optimization with modal mass constraints - results and discussions}

Table 3 shows the distribution of the modal masses extracted from the HF FE model, calculated using Eq. (12). It can be observed that the first six modes are associated to $88 \%$ of the total inertia of the blade. This proves that considering the first six modes provides a good approximation of the overall blade dynamics. With the increase of the number of modes the share of the inertia will not be significantly modified. and the penalty costs for the computation of the ROM would increase..

The optimization process has been performed on the 3D ROM, given its superior performance with respect to the $2 \mathrm{D}$ model. The configurations corresponding to the initial static mass constraint in Eq. (11) have been considered as initial guesses. Figure 10 shows the Pareto Front related to the optimal results as a compromise between the MAC, NF and relative modal mass errors versus the weight ratio for the three objectives. The optimal results with two weight ratios are then compared with the optimal results from the static mass optimization shown in Figure 11.

Figure 11(a) shows the results associated to a weight ratio 1:1:1. The NF error with modal mass objective increases significantly compared to the one provided by the static mass constraint. However, the error with respect to the HF FE model is still within $3 \%$. It is worth observing that the NF error on the $2^{\text {nd }}$ is strongly affected by the use of the modal masses as objectives. The error associated to the MAC overall remains similar $(\sim 8 \%)$ for the first four modes for both the weight ratios (Figure $11 \mathrm{~b}$ ), but it tends to increase for the $5^{\text {th }}$ and $6^{\text {th }}$ modes when the modal mass objective is considered. The modal mass errors with weight ratio of $1: 1: 1$ vary between $2 \%$ and $12 \%$ error on average, and plateau at an average 7\% with the weight ratio of 1:2:3 (Figure $11 \mathrm{~b}$ ). The use of the modal mass objective with the 1:2:3 weight ratio increases the NF error, which rises to $4 \%$ overall. Further rise of the weight ratio values in Obj 3 leads to NF and MAC errors to increase proportionally. The low relative modal mass error means that the amount of effective mass excited in each mode is relatively close between the HF-FE and the 3D ROM [31]. The larger errors in natural 
frequencies and MAC values observed when the modal masses are used as an objective function can be explained by the fact that only $88 \%$ of the actual static mass is considered in the ROM when the first six modes from the HF FE are selected. In principle, it would be possible to redefine the cost function in Eq. (14) by considering both the objective on the total mass and that on the static masses. However, this will imply introducing a fourth weight, thus making the analysis and the representation of the Pareto fronts more complex. Nonetheless, it is worth noticing that the cost function including only the objective function on the static mass (16) achieves very satisfactory results and it therefore adequate for practical applications.

\section{Modes comparison and sensitivity study}

Table 4 shows the comparison of the natural frequencies and relative errors calculated through the Timoshenko beam ROM and the 3D beam frame against the HF FE model for the first six modes. The 3D beam ROM frame has a significantly lower relative error compared to the Timoshenko beam ROM model for the last first three modes. The direct comparison between mode shapes from the beam frame and the HF FE model is also shown in Figure 12. The 3D beam frame ROM model is not only able to capture the first few fundamental modes (like the Timoshenko beam model), but also predicts some asymmetrical features of the modes, which can also be observed in the first global flapwise bending mode of the blade. Most importantly, the beam frame model can capture the coupled bending and torsion modes of mode shapes 3, 4 and 5 of the blade, and to some extent also the sixth edge wise mode. The direct number of DOFS in the two ROM and the high-fidelity model is in the ratio of 1:2:1350. The beam frame ROM is therefore 600 times less large than the FE model, and only double the size of the traditional Timoshenko beam approach.

The previous results have shown that the 3D beam frame ROM model represents a robust approximation of the modal behavior of the twisted blade, restricted in this work to the first six modes. The deviations of the natural frequencies are used to show the perturbation created by the geometrical and material mistuning. The main source of uncertainty for the blade comes appears to come from the joints and imperfections due to damage. A sensitivity study using the 3D ROM model is carried out with two types of perturbation. The first consists in the lumped mass approach, a standard procedure in industry to represent the uncertainty from the geometrical mistuning. The lumped masses are usually placed in the points corresponding to the highest modal displacements of several models (especially if close), so that the uncertainty can be successfully propagated in a specific interested frequency bandwidth covered by the modes considered. In this case, a point mass without inertial is lumped at the tip of the blade on both sides separately, as shown in Figure 13. In the second perturbation case, the Young's modulus and the density are used as separate perturbation parameters. The sensitivity of the high-fidelity FE model and the $3 \mathrm{D}$ beam frame ROM is directly compared for the two perturbation cases.

Figures 14 and 15 show the sensitivity of natural frequencies for the HF FE and 3D beam frame models when a single mass is attached on the two locations of the tip. Both the high fidelity and the ROM model show consistent and similar trends, especially for the modes showing higher sensitivity like the 3rd (Figure 14) and the $1^{\text {st }}$, 2nd and 4th (Figure 15). The results confirm that the 3D frame ROM model can follow the variation of the dynamic behavior of the blade when subject to geometry mistuning. The relative differences in NF for the two models are separately evaluated at ten sampling points from $-2 \%$ to $2 \%$. The average values for the six modes are shown in Figures 14(c) and 15(c). For the lumped mass located in the first position (Figure 13(a)) the average error tend to be around $30 \%$, with the largest discrepancy of $48 \%$ associated to the 5 th mode. It is worth noticing that the second flapwise bending mode ( $3 \mathrm{rd}$ mode) has an error against the high-fidelity model of $8 \%$ only. The situation is different when 
the mass is lumped at the second location (Figure 13(b)), for which the average error for the first four modes is around $20 \%$, and the highest discrepancy appears in the last two modes $(45 \%$ and $90 \%)$. The likely reason behind the presence of high percentage errors can be attributed to the limited number of beam elements representing the frame model. Three elements per span and one element per width are sufficient to capture the modal behavior of the high fidelity blade with no perturbation, but they appear to be not sufficient lumped masses are attached, because of the local changes in dynamic stiffness that involve membrane/flexural/torsional displacements that are difficult to include in a single Timoshenko beam element. Additional calculations indicate that by increasing the number of beam frames one would improve the representation of the coupled mode shapes, especially on 45 and $6^{\text {th }}$ mode, as more parameters can be used for the calibration. The beam frame with a higher number of beams could reduce the error caused by the presence of the lumped masses, although this would significantly increase the computational costs of the development of the ROM.

Figures 16 and 17 illustrate the sensitivity of the natural frequencies in the HF FE model and 3D beam frame models when the material properties are varied. Similarly to the lumped mass case, the sensitivity trends between the full scale twisted blade and the frame model are very close. The most significant feature of these sensitivity analysis is however the extremely low discrepancy between the high fidelity and the ROM, with average errors around $0.2 \%$ for the Young's modulus case, and $0.15 \%$ for the density. No specific mode in particular seems to be affected by the uncertainty associated to the material properties. These results suggest that the 3D frame ROM model can be efficiently used during parametric analysis related to the blade design when different materials need to be evaluated.

\section{Conclusions}

The main objective of this work is the development of a parametric ROM representing a typical aeroengine blade. The ROM consists in a simplified structural layout that provides an equivalent dynamic behavior of the full-scale blade for the first six modes. The ROM concept makes use of 2D and 3D EulerBernoulli frames, whose beam elements are connected at locations that correspond to the experimental measurement points of frequency response functions on a real blade. The ROM frame concept can represent coupled flexural/torsional mode shapes, the relevant natural frequencies and the modal masses of the baseline reference case. A high fidelity FE model representing a real blade was employed as the aforementioned reference. The initial configurations of the ROM frames have been identified by exploiting the orthogonality properties of the modes obtained from the high-fidelity FE model. The parameters defining the frame configurations have been further calibrated via an optimization process based on simulated annealing with stochastic tunneling. The cost functions of the optimization process are expressed by linear combinations of relative errors associated to the natural frequencies, the individual $\mathrm{MAC}$, and static or modal masses.

The parametric 3D ROM beam frame model captures in an effective way the dynamic behavior of the fan blade. The optimized 3D beam frame greatly outperforms the $2 \mathrm{D}$ case in terms of the MAC error $(8 \%$ versus $30 \%$ ), providing a better description of the coupling existing between in-plane deformations and out-of-plane torsion. However, updating the $2 \mathrm{D}$ beam frame model via the optimization procedure is 4 times less computationally expensive than for the 3D ROM. The errors on the estimation of the natural frequencies are minimized when the cost function comprises the overall static mass as objective, while the objective expressed in terms of modal masses leads to larger discrepancies with respect to the reference case. However, the error associated to the MAC objective remains almost constant in both cases. When considering the optimal set of results, the relative error on the modal masses averages at $7 \%$, while that associated to the static mass is below $1 \%$. 
The frame ROM proposed in this paper yields considerably more accurate results than ROMs based on an equivalent Timoshenko beams, especially when there is the need to represent coupled bending and torsion modes, chord-wise motion and edge-wise flap, while retaining a high computational efficiency. Sensitivity analysis on the natural frequencies of the 3D frame ROM and the high fidelity model on lumped masses and the blade material properties show that the ROM approach proposed in this paper can predict the global variation trends of the twisted blade. The discrepancies between natural frequencies predicted by the ROM model and the full-scale blade in mass sensitivity tend to be more significant than the ones observed when the material properties of the blade are used as sensitivity parameters. The results show also that the frame ROM model can be used effectively in low-cost computational parametric analysis of full-scale mistuned bladed discs with uncertainties from material parameters, uncertainties from the joint and geometrical mistuning represented by lumped masses or springs.

\section{Acknowledgements}

The authors would like to acknowledge the support of Rolls-Royce plc for the support of this research through the Composites University Technology Centre (UTC) at the University of Bristol, UK. The Authors also acknowledge the support from the Strategic Investment in Low carbon Engine Technology (SILOET) programme supported by Rolls-Royce plc \& the Technology Strategy Board (TSB). JY is also grateful to the China Scholarship Council for the support. The authors also thank the Reviewers for their useful suggestions.

\section{Reference}

[1] A. Sinha, B. Hall, B. Cassenti, G. Hilbert, Vibratory parameters of blades from coordinate measurement machine data, Journal of Turbomachinery, 130 (2008) 011013.

[2] P. Vargiu, C.M. Firrone, S. Zucca, M.M. Gola, A reduced order model based on sector mistuning for the dynamic analysis of mistuned bladed disks, International Journal of Mechanical Sciences, 53 (2011) 639-646.

[3] D.J. Ewins, The effects of detuning upon the forced vibrations of bladed disks, Journal of Sound and Vibration, 9 (1969) 65-79.

[4] C.H. Hodges, Confinement of vibration by structural irregularity, Journal of Sound and Vibration, 82 (1982) 411-424.

[5] C. Hodges, J. Woodhouse, Vibration isolation from irregularity in a nearly periodic structure: Theory and measurements, The Journal of the Acoustical Society of America, 74 (1983) 894.

[6] C. Pierre, Mode localization and eigenvalue loci veering phenomena in disordered structures, Journal of Sound and Vibration, 126 (1988) 485-502.

[7] M.P. Castanier, C. Pierre, Modeling and Analysis of Mistuned Bladed Disk Vibration: Current Status and Emerging Directions, Journal of Propulsion and Power, 22 (2006) 384-396.

[8] J. MacBain, P. Whaley, Maximum resonant response of mistuned bladed disks, Journal of Vibration Acoustics Stress and Reliability in Design, 106 (1984) 218.

[9] D. Whitehead, The maximum factor by which forced vibration of blades can increase due to mistuning, Journal of Engineering for Gas turbines and Power, 120 (1998) 115-119.

[10] M. Imregun, D. Ewins, Aeroelastic vibration analysis of tuned and mistuned bladed systems, in: Proceedings of the Second Symposium on Unsteady Aerodynamics of Turbomachines and Propellers, Cambridge, UK, 1984.

[11] R. Rzadkowski, The general model of free vibrations of mistuned bladed discs, part i: Theory, Journal of Sound and Vibration, 173 (1994) 377-393. 
[12] H. Irretier, Spectral Analysis of Mistuned Bladed Disk Assemblies by Component Mode Synthesis, in: D.J.E.A.V. Srinivasan (Ed.) Vibrations of Bladed Disk Assemblies, American Society of Mechanical Engineers, New York, 1983.

[13] G. Óttarsson, Dynamic modeling and vibration analysis of mistuned bladed disks, in, University of Michigan, 1994.

[14] M.-T. Yang, J. Griffin, A reduced order approach for the vibration of mistuned bladed disk assemblies, Journal of Engineering for Gas turbines and Power, 119 (1997) 161-167.

[15] W. Joo, K. Yee, D.-H. Lee, W. HURTY, Dynamic analysis of structural systems using component modes, AIAA journal, 3 (1965) 678-685.

[16] M. Petyt, Introduction to finite element vibration analysis, Cambridge university press, 2010.

[17] A. Butland, P. Avitabile, A reduced order, test verified component mode synthesis approach for system modeling applications, Mechanical Systems and Signal Processing, 24 (2010) 904-921.

[18] M.-T. Yang, J. Griffin, A reduced-order model of mistuning using a subset of nominal system modes, Journal of Engineering for Gas turbines and Power, 123 (2001) 893-900.

[19] D.M. Feiner, J. Griffin, A fundamental model of mistuning for a single family of modes, Journal of turbomachinery, 124 (2002) 597-605.

[20] S.-H. Lim, R. Bladh, M.P. Castanier, C. Pierre, Compact, Generalized Component Mode Mistuning Representation for Modeling Bladed Disk Vibration, AIAA Journal, 45 (2007) 2285-2298.

[21] M. Al-Aghbari, F. Scarpa, W. Staszewski, On the orthogonal wavelet transform for model reduction/synthesis of structures, Journal of Sound Vibration, 254 (2002) 805-817.

[22] M. Friswell, S. Garvey, J. Penny, Model reduction using dynamic and iterated IRS techniques, Journal of sound and vibration, 186 (1995) 311-323.

[23] J.M. Brown, J. Slater, R.V. Grandhi, Probabilistic analysis of geometric uncertainty effects on blade modal response, in: ASME Turbo Expo 2003, collocated with the 2003 International Joint Power Generation Conference, American Society of Mechanical Engineers, 2003, pp. 247-255.

[24] V.E. Garzon, D.L. Darmofal, Impact of geometric variability on axial compressor performance, in: ASME Turbo Expo 2003, collocated with the 2003 International Joint Power Generation Conference, American Society of Mechanical Engineers, 2003, pp. 1199-1213.

[25] D.J. Malcolm, D.L. Laird, Modeling of blades as equivalent beams for aeroelastic analysis, in: 2003 ASME Wind Energy Symposium AIAA/ASME, 2003, pp. 293-303.

[26] A.M.T. Hach, P. Co, M. Carleton University. Dissertation. Engineering, Aerospace, Aeroelastic Analysis of a Turbine Blade Using a Reduced-order Model, Carleton University (Canada), 2007.

[27] S.K. Sinha, K.E. Turner, Natural frequencies of a pre-twisted blade in a centrifugal force field, Journal of Sound and Vibration, 330 (2011) 2655-2681.

[28] T.L. Zhu, The vibrations of pre-twisted rotating Timoshenko beams by the Rayleigh-Ritz method, Computational Mechanics, 47 (2010) 395-408.

[29] S.K. Campus, Effects of damping and varying contact area at blade-disk joints in forced response analysis of bladed disk assemblies, Journal of turbomachinery, 128 (2006) 403.

[30] M.H. Richardson, C. Jamestown, Modal mass, stiffness and damping, in: Vibrant Technology, Inc., Jamestown, CA, 2000, pp. 1-5.

[31] A. Piersol, T. Paez, Harris' Shock and Vibration Handbook, Mcgraw-hill, 2009.

[32] J.S. Przemieniecki, Theory of matrix structural analysis, Dover Publications, 1985.

[33] D. Fotsch, D. Ewins, Application of MAC in the frequency domain, ROLLS ROYCE PLC-REPORT-PNR, (2000).

[34] F. M, G. SD, P. JET, Model reduction using dynamic and iterated IRS techniques, Journal of Sound and 
Vibration, 186 (1995) 311-323.

[35] U. Lee, Dynamic continuum modeling of beamlike space structures using finite-element matrices, AIAA journal, 28 (1990) 725-731.

[36] T. Karaağaçlı, E.N. Yıldız, H. Nevzat Özgüven, A new method to determine dynamically equivalent finite element models of aircraft structures from modal test data, Mechanical Systems and Signal Processing, 31 (2012) 94-108.

[37] M. Friswell, J.E. Mottershead, Finite element model updating in structural dynamics, Springer, 1995. [38] S. Lin, Modal Mass Computation Based on ANSYS Finite Element Analysis, Missiles and Space Vehicles, 3 (2011) 013.

[39] T. Karaağaçlı, E.N. Yıldız, H. Nevzat Özgüven, A new method to determine dynamically equivalent finite element models of aircraft structures from modal test data, Mechanical Systems and Signal Processing, (2012).

[40] R. Levin, N. Lieven, Dynamic finite element model updating using simulated annealing and genetic algorithms, Mechanical Systems and Signal Processing, 12 (1998) 91-120.

[41] W. Wenzel, K. Hamacher, Stochastic tunneling approach for global minimization of complex potential energy landscapes, Physical Review Letters, 82 (1999) 3003-3007.

\section{Figures and Tables}

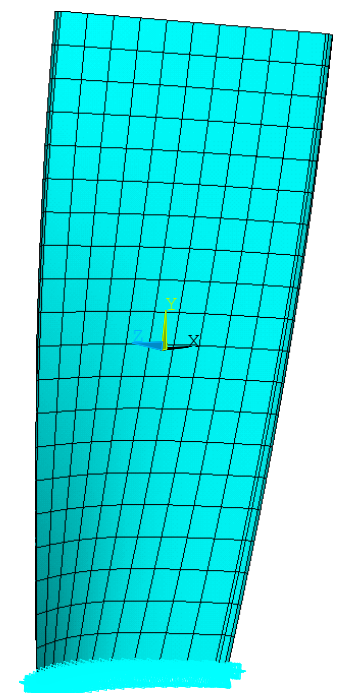

Figure1 Finite Element blade model used as reference. 


\begin{tabular}{|c|c|c|c|}
\hline NF & Chord wise (mm) & Span-wise (mm) & Out of plane (mm) \\
\hline $\begin{array}{l}\text { Mode1 } \\
\omega 1\end{array}$ & Min to Max 0 to 14 & Min to Max -3 to 7 & Min to Max 0 to 37 \\
\hline $\begin{array}{l}\text { Mode2 } \\
\omega 2\end{array}$ & Min to Max -1 to 42 & Min to Max -3 to 6 & Min to Max -26 to 28 \\
\hline $\begin{array}{l}\text { Mode3 } \\
\omega 3\end{array}$ & Min to Max -14 to 34 & Min to Max -3 to 3 & Min to Max -23 to 41 \\
\hline $\begin{array}{l}\text { Mode4 } \\
\omega 4\end{array}$ & Min to Max -23 to 31 & Min to Max -4 to 12 & Min to Max -20 to 46 \\
\hline $\begin{array}{l}\text { Mode5 } \\
\omega 5\end{array}$ & \begin{tabular}{|l|} 
Min to Max 0 to 32 \\
\end{tabular} & Min to Max -10 to 6 & Min to Max -25 to 32 \\
\hline $\begin{array}{l}\text { Mode6 } \\
\omega 6\end{array}$ & Min to Max -4 to 35 & Min to Max -3 to 12 & Min to Max -27 to 44 \\
\hline
\end{tabular}

Figure 2 The first six modes of the aeroengine fan blade 


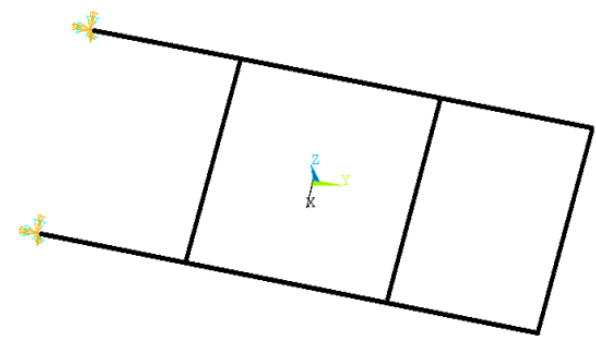

(a)

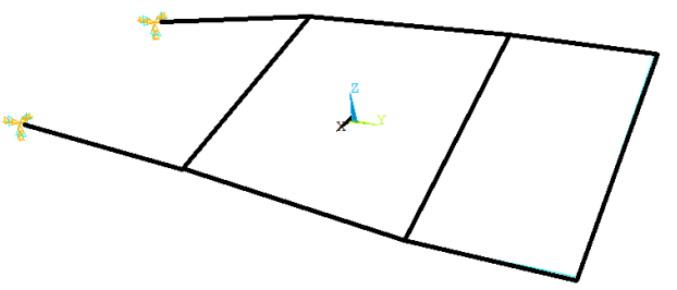

(b)

Figure 3 (a) Deformed 2D frame (b) Deformed 3D frame
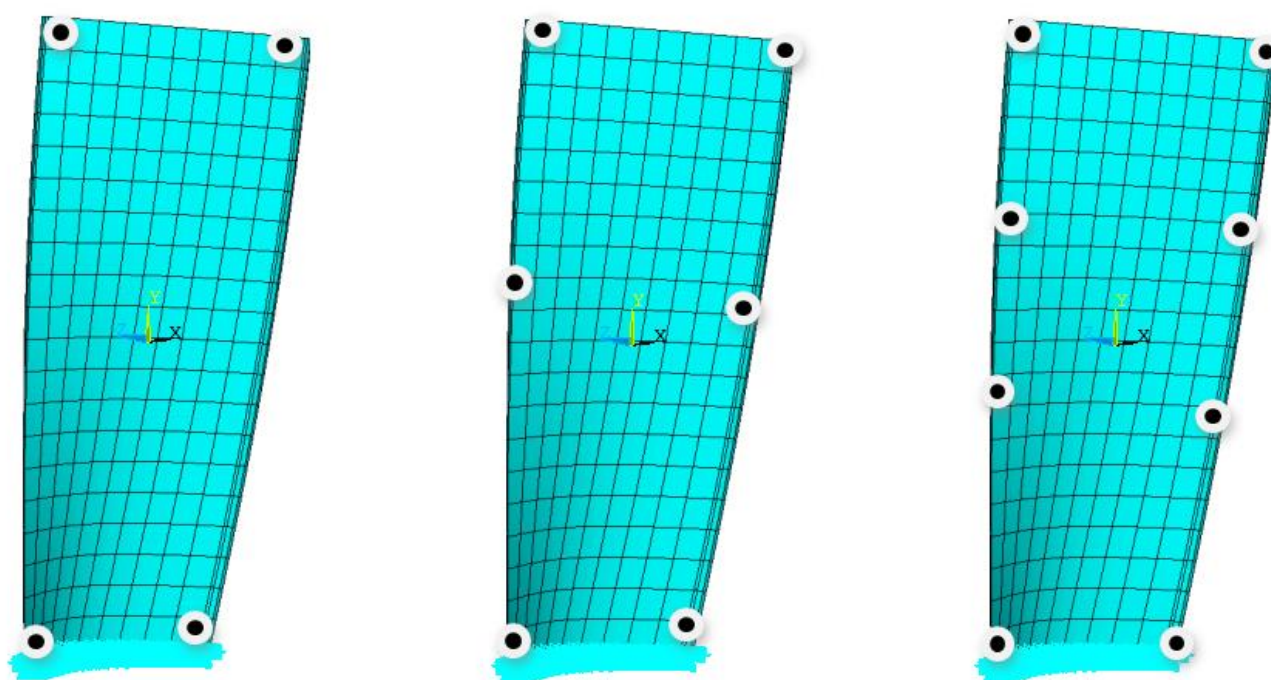

Figure 4 Measuring points on the blade corresponding to the nodes on the ROMs
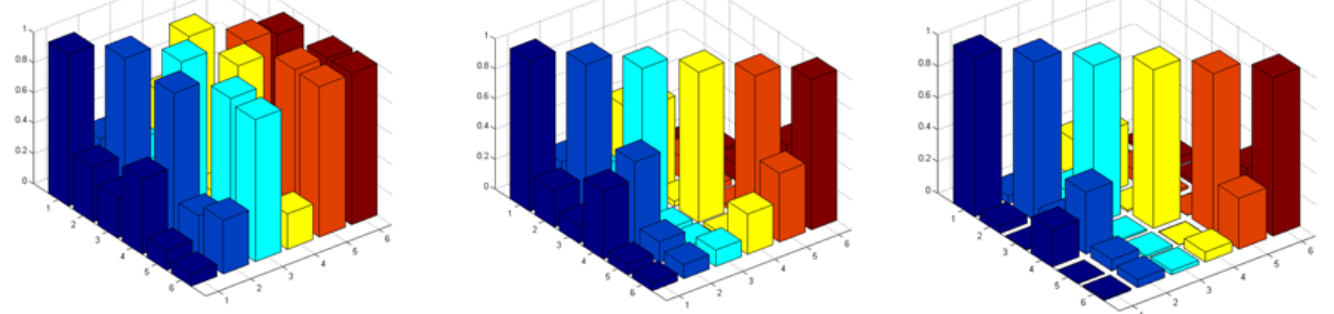

Figure 5 Auto MAC plots for the first six modes with ROMs having 4, 6 and 8 nodes 


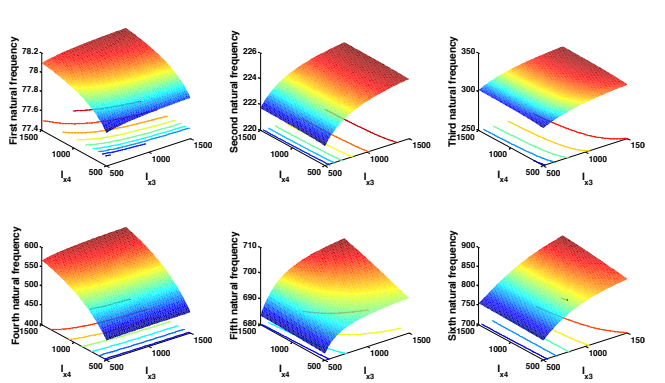

(a)
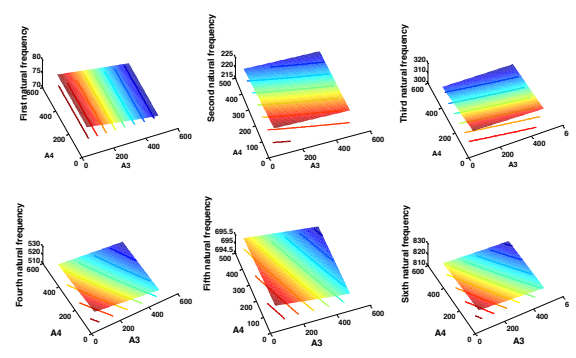

(b)

Figure 6 (a) Surface responses of NFs with $\mathrm{I}_{\mathrm{x} 3} \mathrm{I}_{\mathrm{x} 4}$ (b) Surface responses of NFs with $A_{3} A_{4}$

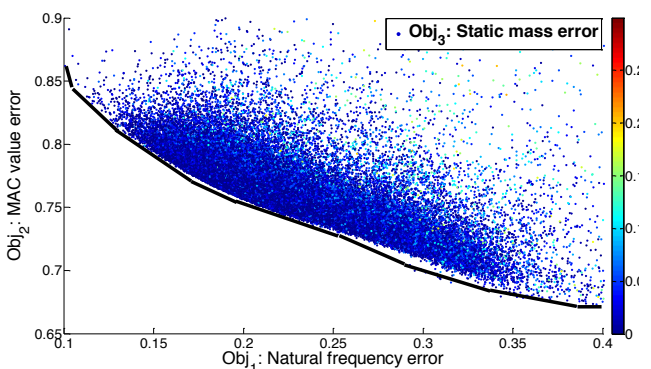

(a)

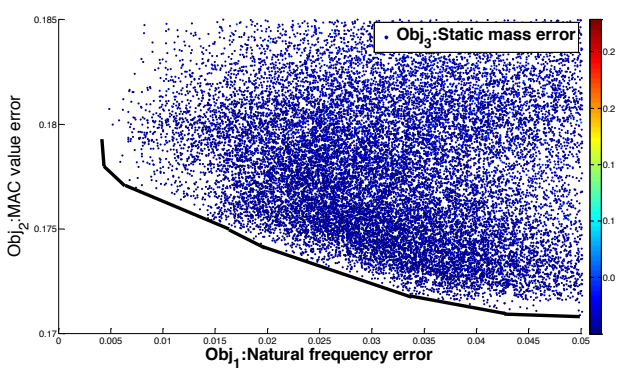

(b)

Figure 7(a) Pareto front plots for 2D frame (b) Pareto front plots for 3D frame
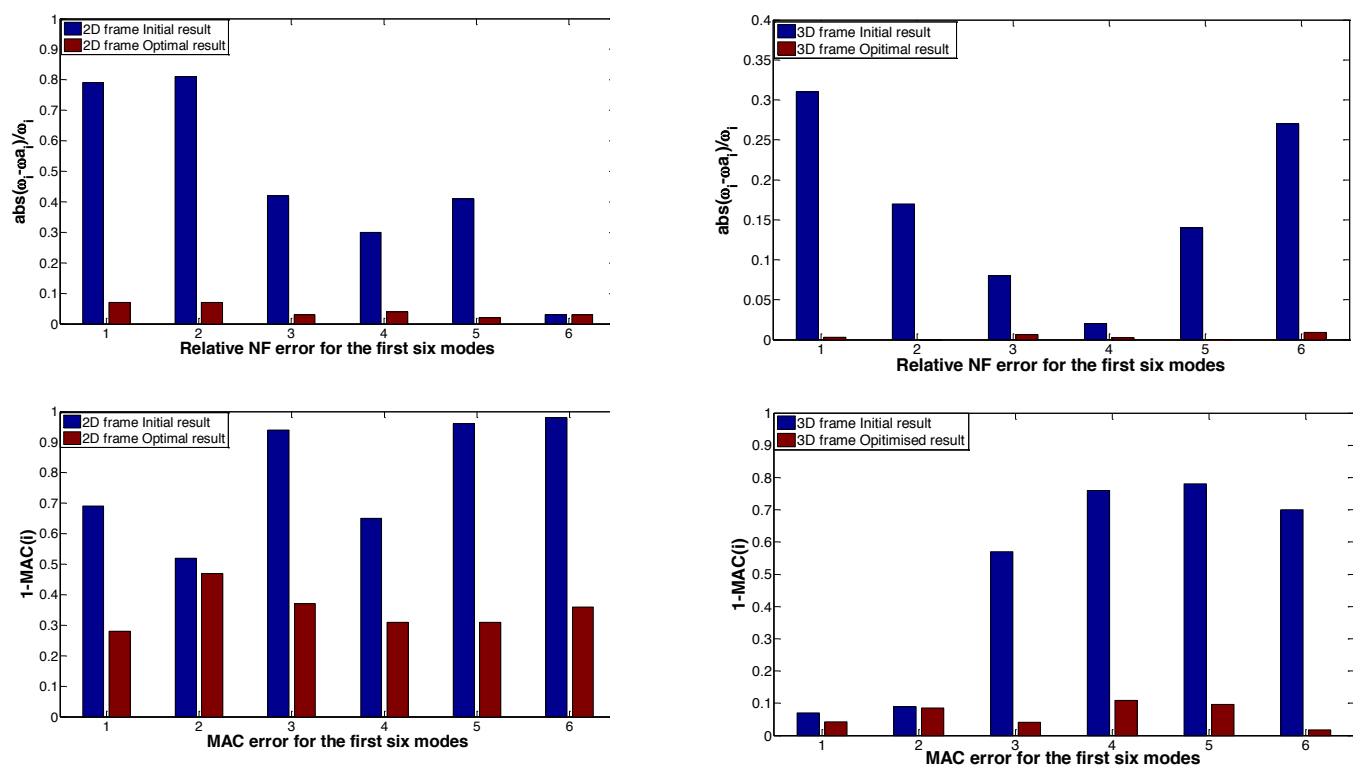
(a)

(b)

Figure 8 From top to bottom (a)NF and MAC error for the 2D frame with weight ratio of 2:2:1 (b)NF and MAC error for the 3D frame with weight ratio of 2:7:1

(a)

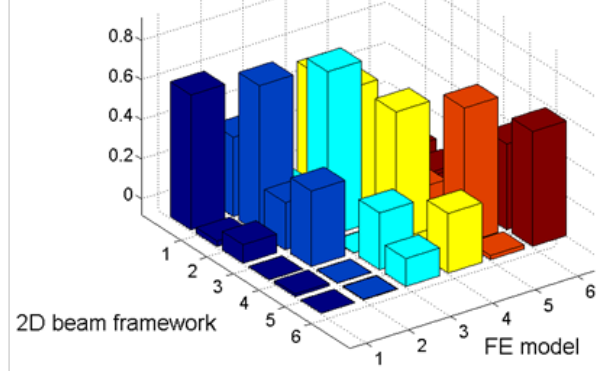

(b)

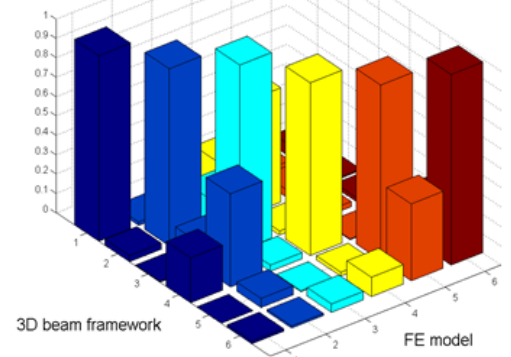

Figure 9 MAC plots for the (a) 2D beam frame and (b) 3D ROM beam frame

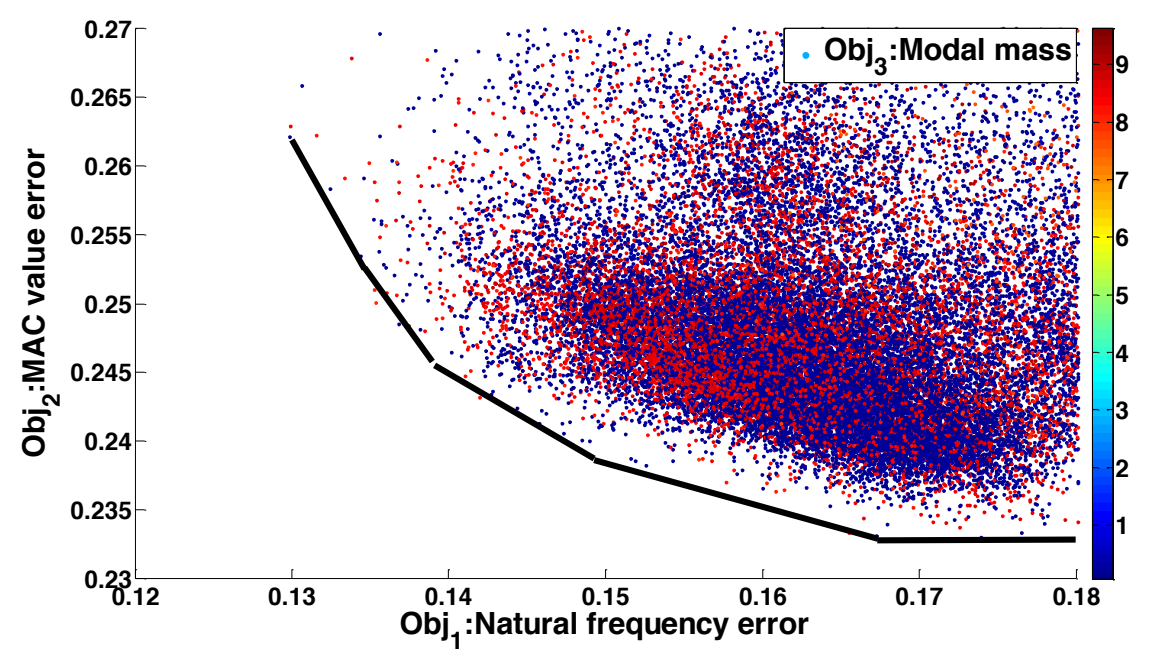

Figure 10 Pareto front plots for 3D ROM frame with modal mass objective 

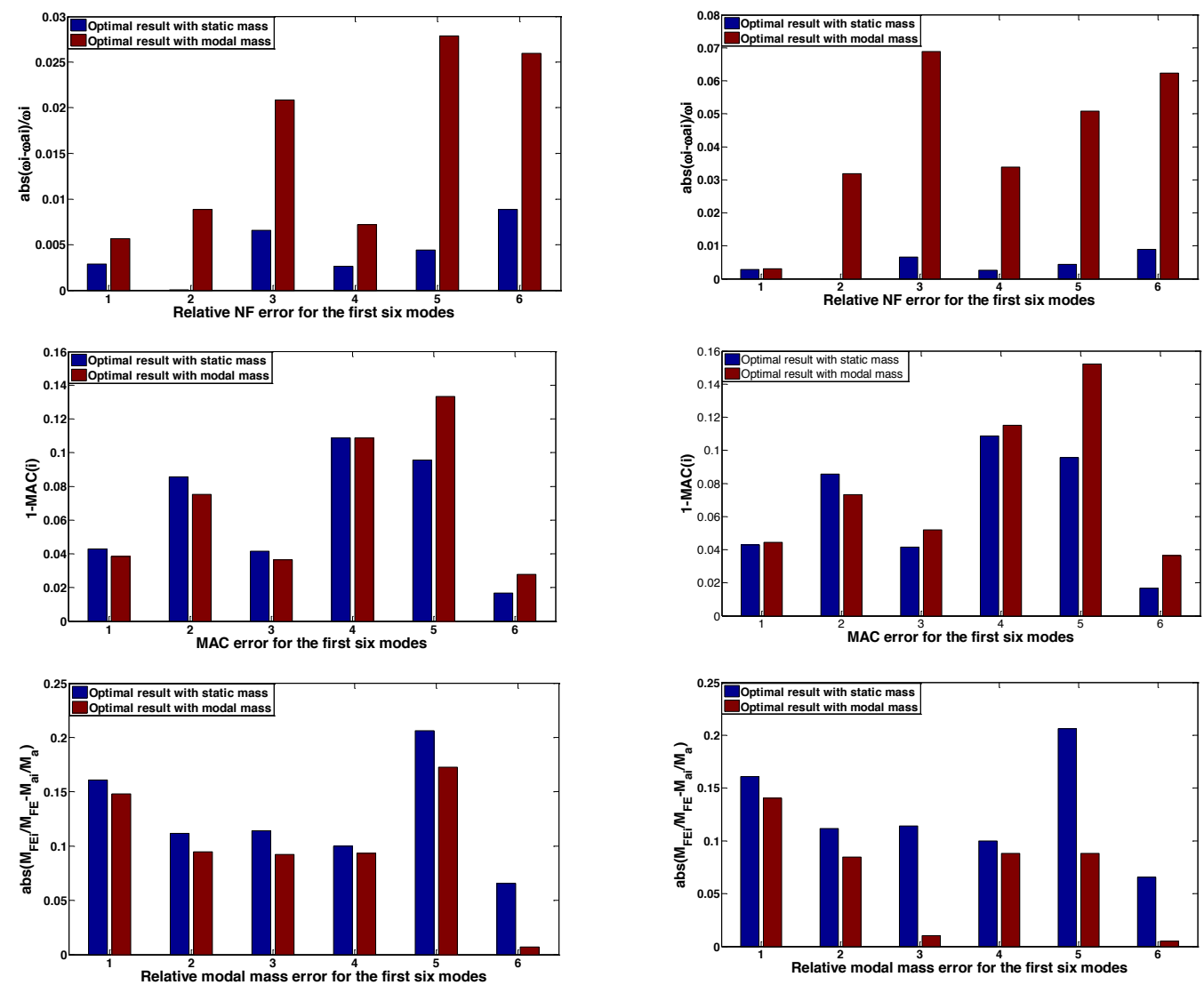

(a)

(b)

Figure 11. From top to bottom: NF, MAC, modal mass errors for 3D frame with (a) weight ratio $1: 1: 1$, and (b) weight ratio $1: 2: 3$ 


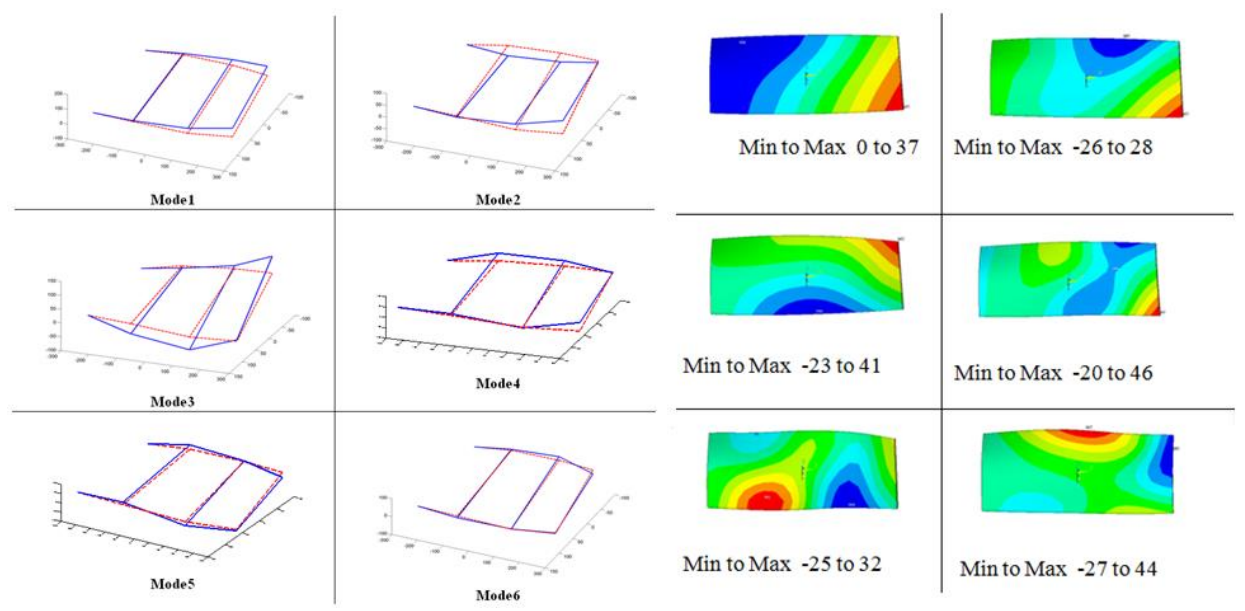

Figure 12 Comparison of the mode shapes of the 3D beam frame and the HF models (out of plane) Red dashed line: original shape Blue line: deformed shape

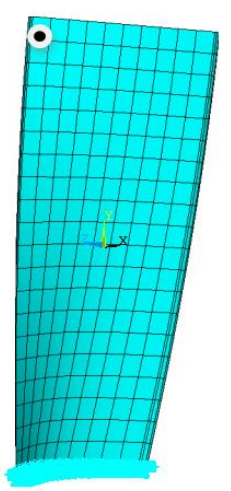

(a)

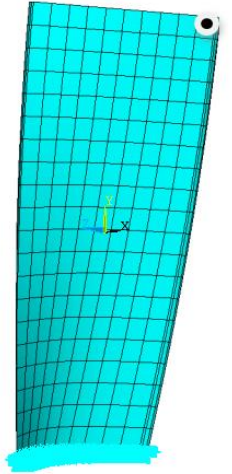

(b)

Figure 13 Locations to lump the point masses (the blade point within the white circle) 


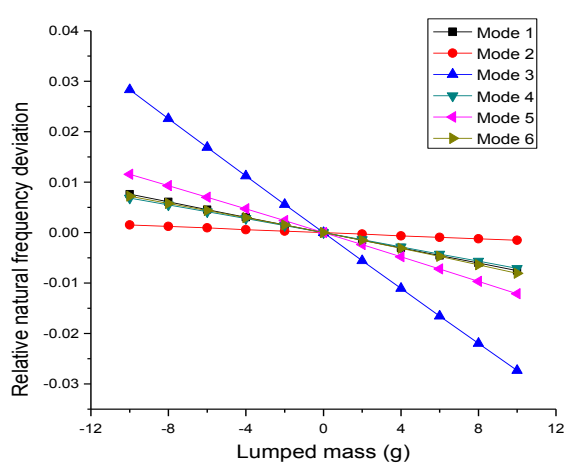

(a)

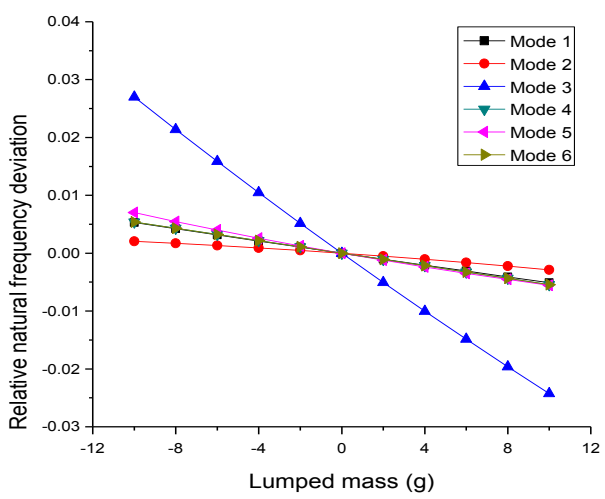

(b)

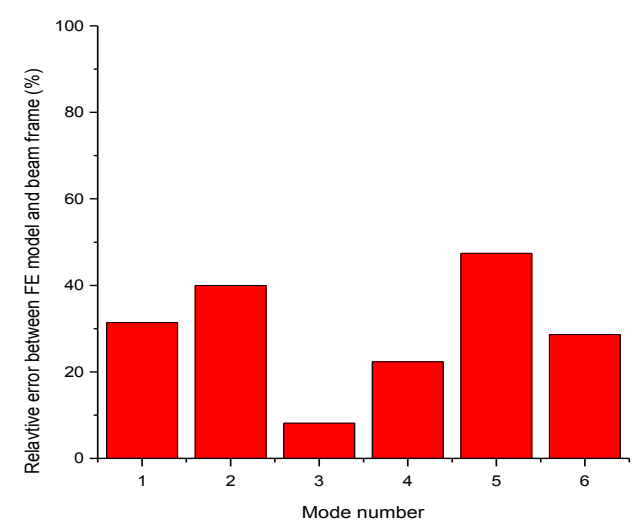

(c)

Figure 14 Sensitivity study with the lumped mass approach in the position 1 (a) HF-FE model (b) 3D Beam frame ROM (c) Relative error 


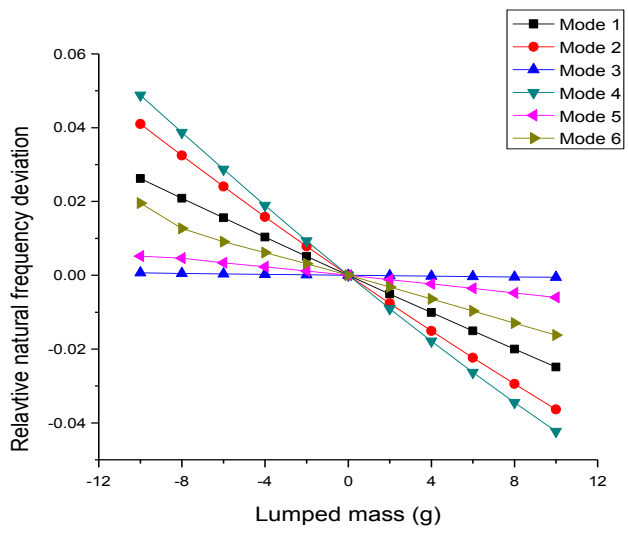

(a)

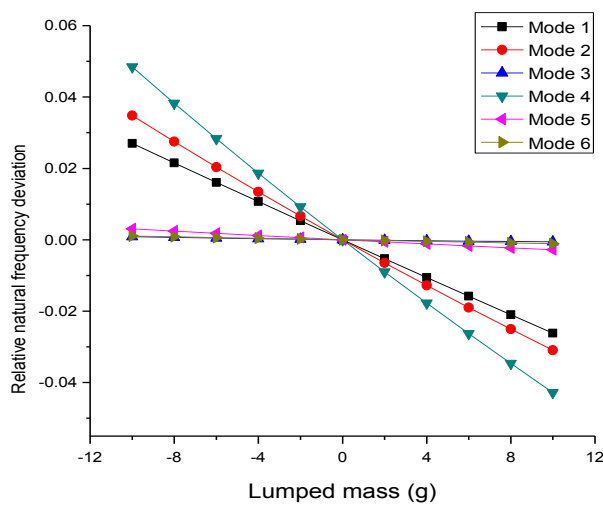

(b)

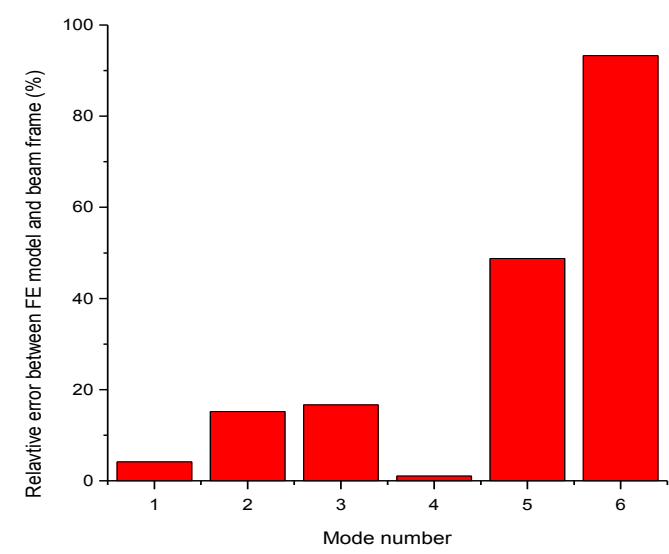

(c)

Figure 15 Sensitivity study with lumped mass approach in position 2 FE model (b) Beam frame (c) Relative error 


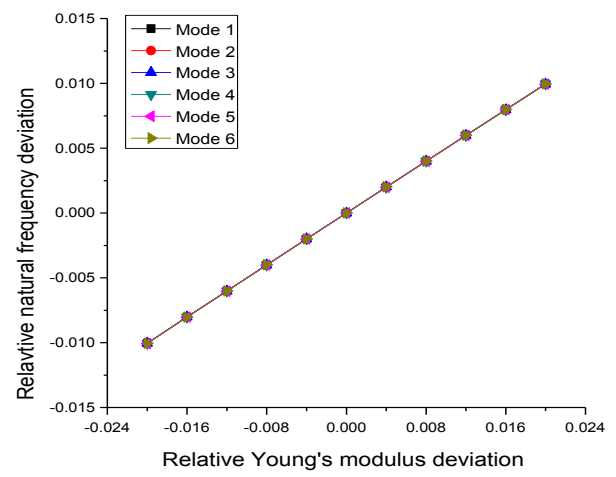

(a)

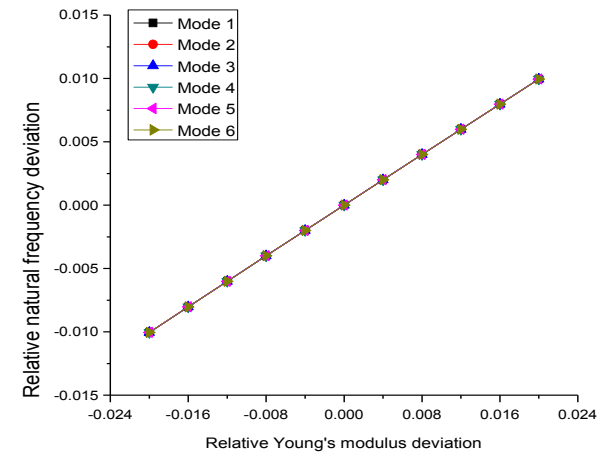

(b)

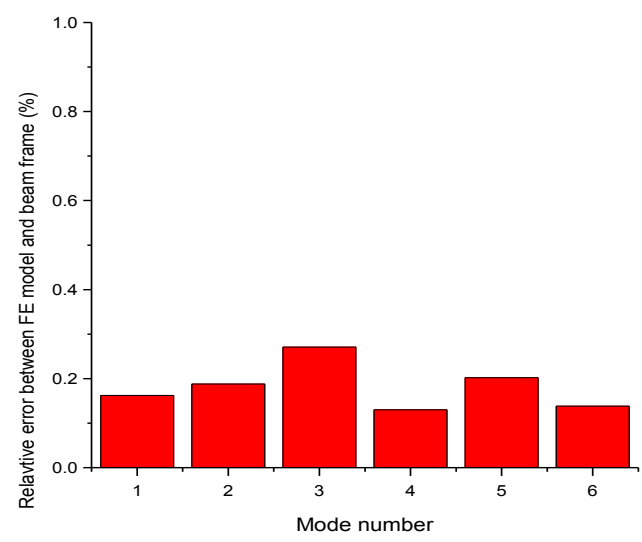

(c)

Figure 16 Sensitivity study with Young's Modulus perturbation (a) FE model (b) Beam frame (c) Relative error 


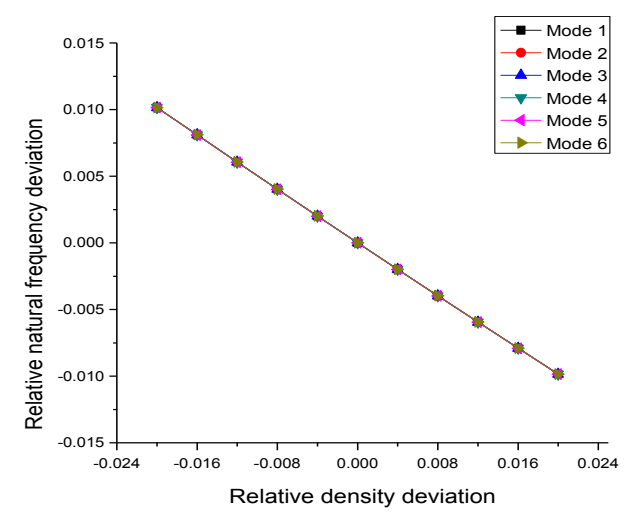

(a)

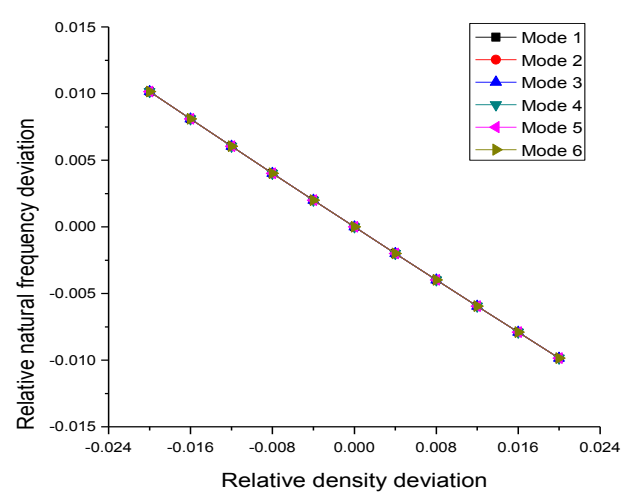

(b)

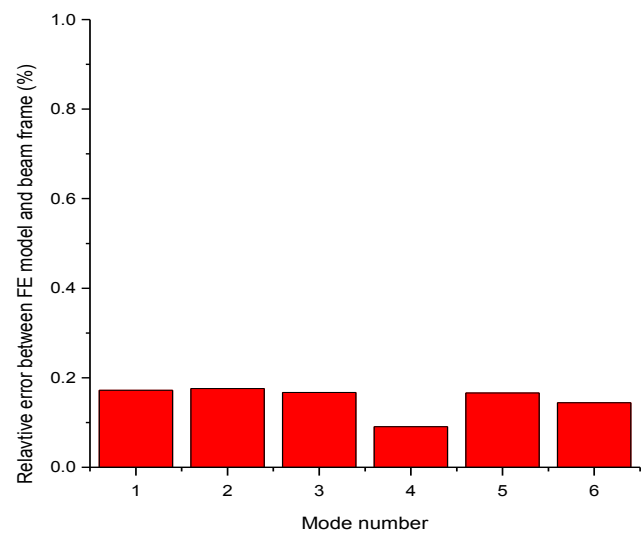

(c)

Figure 17 Sensitivity study with density perturbation

(a) FE model (b) Beam frame (c) Relative error

Table 1 Natural frequency comparison between the beam model and HF FE model

\begin{tabular}{|c|c|c|c|}
\hline Mode Number & $\begin{array}{c}\text { Timoshenko beam } \\
\left(\omega_{i}^{a} / \omega_{1}\right)\end{array}$ & $\begin{array}{c}\text { High Fidelity FE model } \\
\left(\omega_{i} / \omega_{1}\right)\end{array}$ & Error (\%) \\
\hline 1 & 0.92 & 1.00 & 8.20 \\
\hline 2 & 3.25 & 2.90 & 11.50 \\
\hline 3 & 4.24 & 4.05 & 5.20 \\
\hline 4 & 11.51 & 6.87 & 67.50 \\
\hline 5 & 11.88 & 8.83 & 34.50 \\
\hline 6 & 17.15 & 10.56 & 62.30 \\
\hline
\end{tabular}


Table 2 Mode shape of the Timoshenko beam ROM model

\begin{tabular}{|c|l|}
\hline Mode Number & \multicolumn{1}{c|}{ Mode shape } \\
\hline 1 & First flap wise bending \\
\hline 2 & First Torsion \\
\hline 3 & Second flap wise bending \\
\hline 4 & Third Flap wise bending \\
\hline 5 & Second Torsion \\
\hline 6 & First chord wise bending \\
\hline
\end{tabular}

Table 3 Relative modal mass for first six modes in the HF FE model

\begin{tabular}{|c|c|}
\hline \multicolumn{1}{|c|}{ Mode } & Modal mass against static mass \\
\hline $\mathbf{1}$ & $17 \%$ \\
\hline $\mathbf{2}$ & $12 \%$ \\
\hline $\mathbf{3}$ & $14 \%$ \\
\hline $\mathbf{4}$ & $11 \%$ \\
\hline $\mathbf{5}$ & $22 \%$ \\
\hline $\mathbf{6}$ & $12 \%$ \\
\hline
\end{tabular}

Table 4 Natural frequency comparison among HF FE model, beam model and beam frame

\begin{tabular}{|c|c|c|c|c|c|}
\hline \multirow{2}{*}{$\begin{array}{l}\text { Mode } \\
\text { Number }\end{array}$} & \multirow{2}{*}{$\begin{array}{l}\text { High Fidelity } \\
\text { FE model } \\
\left(\omega_{i} / \omega_{1}\right)\end{array}$} & \multicolumn{2}{|c|}{ Timoshenko beam } & \multicolumn{2}{|c|}{ 3D Beam frame } \\
\hline & & $\omega_{i}^{a} / \omega_{1}$ & $\begin{array}{l}\text { Erro } \\
\mathrm{r}(\%)\end{array}$ & $\omega_{i}^{a} / \omega_{1}$ & $\begin{array}{l}\text { Error } \\
(\%)\end{array}$ \\
\hline 1 & 1.00 & 0.92 & 8.20 & 0.99 & 0.13 \\
\hline 2 & 2.90 & 3.25 & $\begin{array}{l}11.5 \\
0\end{array}$ & 2.90 & 0.01 \\
\hline 3 & 4.05 & 4.24 & 5.20 & 4.05 & 0.01 \\
\hline 4 & 6.87 & 11.51 & $\begin{array}{l}67.5 \\
0\end{array}$ & 6.87 & 0.02 \\
\hline 5 & 8.83 & 11.88 & $\begin{array}{l}34.5 \\
0 \\
\end{array}$ & 8.82 & 0.12 \\
\hline 6 & 10.56 & 17.15 & $\begin{array}{l}62.3 \\
0\end{array}$ & 10.43 & 0.14 \\
\hline
\end{tabular}

